\title{
Breaking the Doping Limit in Silicon by Deep Impurities
}

\author{
Mao Wang,,${ }^{1,2, *}$ A. Debernardi, ${ }^{3,+}$ Y. Berencén, ${ }^{1}$ R. Heller, ${ }^{1}$ Chi Xu,,${ }^{1,2}$ Ye Yuan, ${ }^{1,4}$ Yufang Xie,,${ }^{1,2}$ \\ R. Böttger, ${ }^{1}$ L. Rebohle, ${ }^{1}$ W. Skorupa,${ }^{1}$ M. Helm,,${ }^{1,2}$ S. Prucnal, ${ }^{1}$ and Shengqiang Zhou ${ }^{1, \dagger}$ \\ ${ }^{1}$ Helmholtz-Zentrum Dresden-Rossendorf, Institute of Ion Beam Physics and Materials Research, Bautzner \\ Landstrasse 400, 01328 Dresden, Germany \\ ${ }^{2}$ Technische Universität Dresden, 01062 Dresden, Germany \\ ${ }^{3}$ CNR-IMM, sede Agrate Brianza, via Olivetti 2, I-20864 Agrate Brianza, Italy \\ ${ }^{4}$ Physical Science and Engineering Division (PSE), King Abdullah, University of Science and Technology \\ (KAUST), Thuwal 23955-6900, Saudi Arabia
}

(Received 24 September 2018; revised manuscript received 14 January 2019; published 14 May 2019)

\begin{abstract}
$n$-type doping in Si by shallow impurities, such as $\mathrm{P}, \mathrm{As}$, and $\mathrm{Sb}$, exhibits an intrinsic limit due to the Fermi-level pinning via defect complexes at high doping concentrations. Here, we demonstrate that doping $\mathrm{Si}$ with the deep chalcogen donor Te by nonequilibrium processing can exceed this limit and yield higher electron concentrations. In contrast to shallow impurities, the interstitial Te fraction decreases with increasing doping concentration and substitutional Te dimers become the dominant configuration as effective donors, leading to a nonsaturating carrier concentration as well as to an insulator-to-metal transition. First-principles calculations reveal that the Te dimers possess the lowest formation energy and donate two electrons per dimer to the conduction band. These results provide an alternative insight into the physics of deep impurities and lead to a possible solution for the ultrahigh electron concentration needed in today's Si-based nanoelectronics.
\end{abstract}

DOI: 10.1103/PhysRevApplied.11.054039

\section{INTRODUCTION}

The scaling evolution for the future generations of Si-based technology, e.g., Si-based transistors in onchip complementary metal oxide semiconductor (CMOS) devices, requires extremely high free-electron densities (around $10^{21} \mathrm{~cm}^{-3}$ ) to ensure enough free carriers in each transistor $[1,2]$. This density is usually achieved with shallow dopants (e.g., group-V dopants such as $\mathrm{P}$, As, and $\mathrm{Sb})$ due to their low ionization energies $(20-50 \mathrm{meV})$, low diffusivities, and suitable solid solubilities in Si. Current technologies, implantation or molecular beam epitaxy, can achieve doping concentrations greater than the solid solubility limit. However, the free-electron concentrations are still found to saturate at around $5 \times 10^{20} \mathrm{~cm}^{-3}$ [35]. The electrical activation approaches a limit where the additional introduced dopant atoms cease to generate extra free carriers. During the past decades, experimental results [6-8] and first-principles calculations [9-11] were applied to investigate the microscopic nature of the electrical

\footnotetext{
*m.wang@hzdr.de

†s.zhou@hzdr.de

$\$$ Author to whom correspondence should be addressed for first principles calculations: alberto.debernardi@mdm.imm.cnr.it
}

deactivation of donors in $\mathrm{Si}$. The $\mathrm{As}_{n} V(n \leq 4)$ model (clustering around a vacancy surrounded by As atoms) [10-17] and the dimer model (two donor atoms bound to reconfigured $\mathrm{Si}$ with no vacancies) $[18,19]$ are two classical models for electrically deactivating dopant complexes. The latter, however, has been suggested to be dominant at high doping levels $[19,20]$. The donor dimers introduce localized deep-level states in the band gap and compensate the free electrons, preventing high electron concentrations $[18,19]$.

Alternatively, it has been found that deep-level impurities (e.g., chalcogen dopants) with ionization energies of several hundred $\mathrm{meV}$ can also induce free electrons (in excess of $10^{20} \mathrm{~cm}^{-3}$ ) in $\mathrm{Si}$ accompanied with an insulator-to-metal transition (IMT) [21,22]. This result makes chalcogens good prospects to be used for overcoming the doping limit in Si. Particularly, one puzzling observation in chalcogen-doped $\mathrm{Si}$ is that the electron concentration increases linearly with the dopant concentration while the substitutional fraction remains almost constant $[23,24]$. For traditional shallow-level dopants, interstitials, inactive clusters, and precipitates often become more energetically favorable as the dopant concentration increases, e.g., in As-doped Si [25,26], Mn-doped GaAs [27,28], and Mg-doped GaN [29,30]. Therefore, scrutinizing the atomic configuration of chalcogen impurities in $\mathrm{Si}$ is needed to 
understand this anomalous electrical activation behavior as well as the sub-band-gap infrared photoresponse [31-33].

In this paper, we unveil the microscopic origin for the high electron density that can be achieved by chalcogen doping. We use Rutherford backscattering channeling to directly identify the lattice location of $\mathrm{Te}$ in $\mathrm{Si}$ and correlate the Te atomistic configuration with the corresponding electrical properties. It is found that, at higher $\mathrm{Te}$ concentration, the fraction of substitutional Te increases, contrary to the behavior of shallow dopants. By firstprinciples calculations, we find that substitutional $\mathrm{Te}$ dimers are energetically more favorable and drive the occurrence of nonsaturating free-electron densities, leading to an insulator-to-metal transition. We thus propose chalcogen dimers as effective donors in $\mathrm{Si}$ for ultrahigh $n$-type doping.

\section{METHODS}

Ion implantation into an intrinsic Si substrate combined with pulsed laser melting (PLM) is applied to synthesize single-crystalline and epitaxial Te-hyperdoped Si layers with a Te concentration several orders of magnitude above the solid solubility limit. Te impurities tend to diffuse toward both the surface and interior of the substrate during the PLM process [33] (see Fig. 8 in Appendix D). The samples are labeled as Te- $0.25 \% \ldots$ Te- $2.5 \%$, with the numbers standing for the percentage of the Te peak concentration. Rutherford backscattering spectrometry in channeling geometries (RBS-C) measurements are performed using a $1.7 \mathrm{MeV} \mathrm{He}{ }^{+}$beam. The backscattered particles are detected at an angle of $170^{\circ}$ with respect to the incoming beam direction using silicon surface barrier detectors. Angular axial scans around different crystallographic directions are obtained using a two-axis goniometer. The electrical properties of the PLM-treated Te-hyperdoped layers are measured using a commercial Lake Shore Hall Measurement System in a van der Pauw configuration [34]. The structural and electronic calculations in the framework of the density functional theory (DFT) are performed by solving the Kohn-Sham equations through the plane-wave pseudopotential approach implemented in the QUANTUM ESPRESSO (QE) open-source code [35]. Further details about experiments and computations can be found in Appendixes A and B.

\section{RESULTS AND DISCUSSION}

\section{A. Electrical properties of Te-hyperdoped Si layers}

Figure 1(a) shows the carrier concentration of Tehyperdoped Si samples measured by the Hall effect at $300 \mathrm{~K}$ as a function of doping concentration. The carrier concentration shown here is in the range of $2.0 \times 10^{19}$ to $8.3 \times 10^{20} \mathrm{~cm}^{-3}$. Being completely different from previously published results for As-, P-, and Sb-doped Si (a)

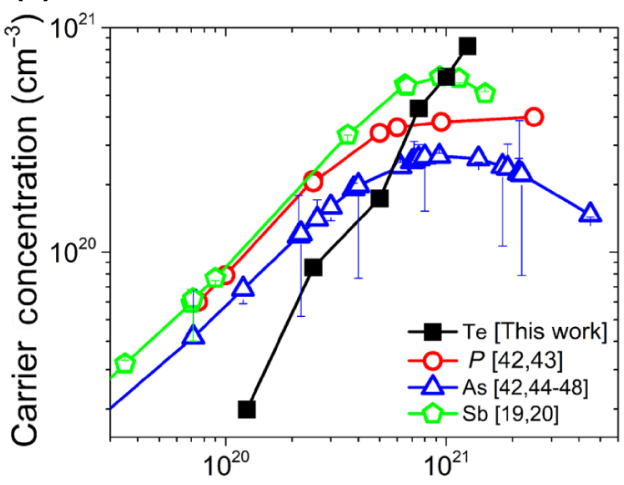

(b) Doping concentration $\left(N_{D}\right)\left(\mathrm{cm}^{-3}\right)$

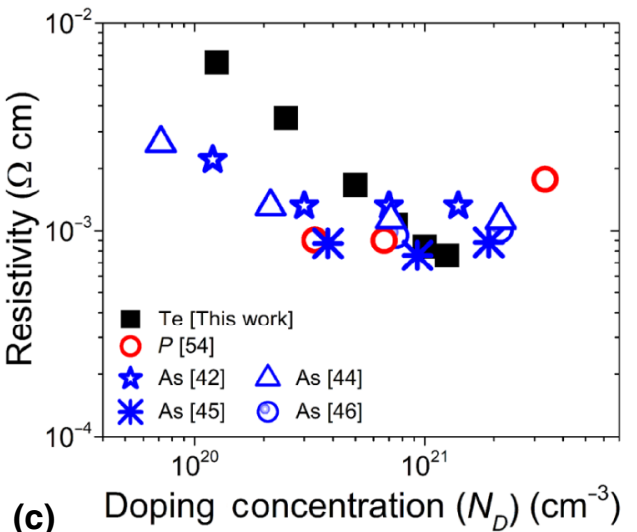

(c) Doping concentration $\left(N_{D}\right)\left(\mathrm{cm}^{-3}\right)$

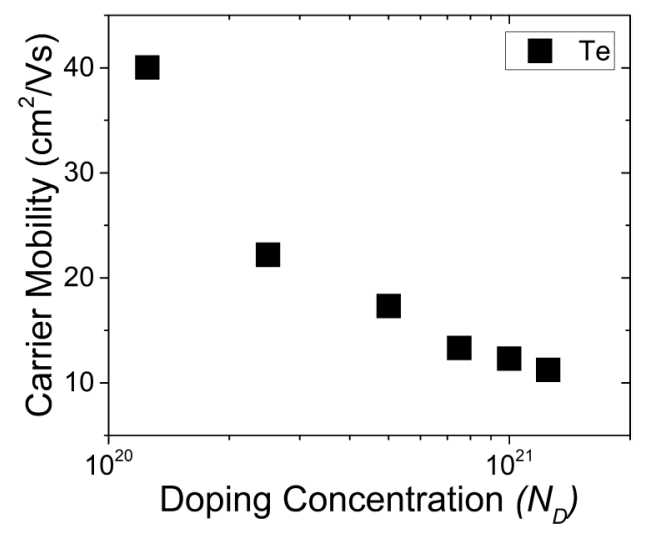

FIG. 1. (a) The carrier concentration of Te-hyperdoped Si samples measured by the Hall effect at $300 \mathrm{~K}$ as a function of doping concentration. The carrier concentration approaches $10^{21} \mathrm{~cm}^{-3}$. Previously published results for As, $\mathrm{P}$, and $\mathrm{Sb}$ heavily doped $\mathrm{Si}$ are also included as references (the error bar represents the difference between reported values). Our results overcome the limit of free-electron concentration (approximately 5-6.5 $\times 10^{20} \mathrm{~cm}^{-3}$ ) obtained by the conventional $n$-type dopants in Si. Saturation in terms of the doping level is not observed within the range of Te concentrations, which are several orders of magnitude above the solid solubility limit of Te in Si. (b) The resistivity of PLM-treated Te-hyperdoped Si samples as a function of doping concentration measured at $300 \mathrm{~K}$. Previous published works for As, $\mathrm{P}$, and $\mathrm{Sb}$ heavily doped $\mathrm{Si}$ are also included as reference. (c) The carrier mobility of PLM-treated Te-hyperdoped Si samples as a function of doping concentration measured at $300 \mathrm{~K}$. 
(a)

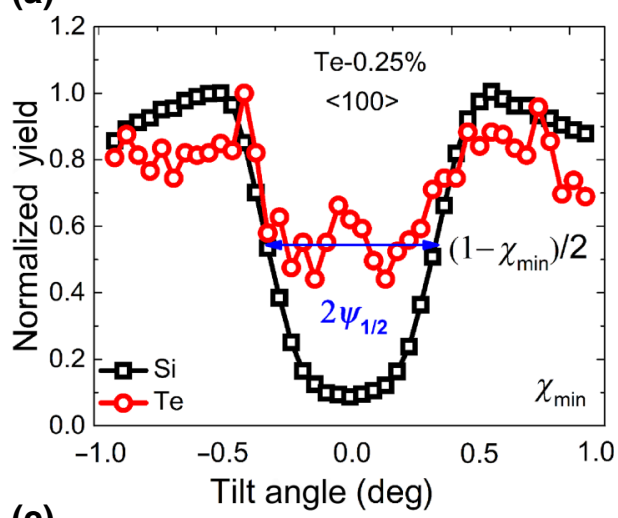

(c)

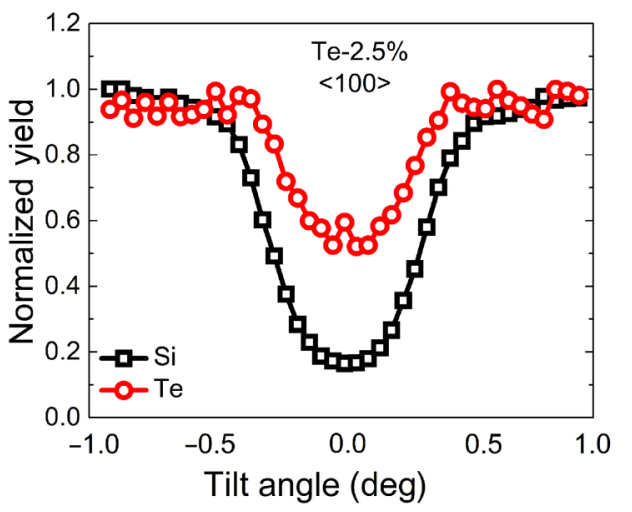

(b)
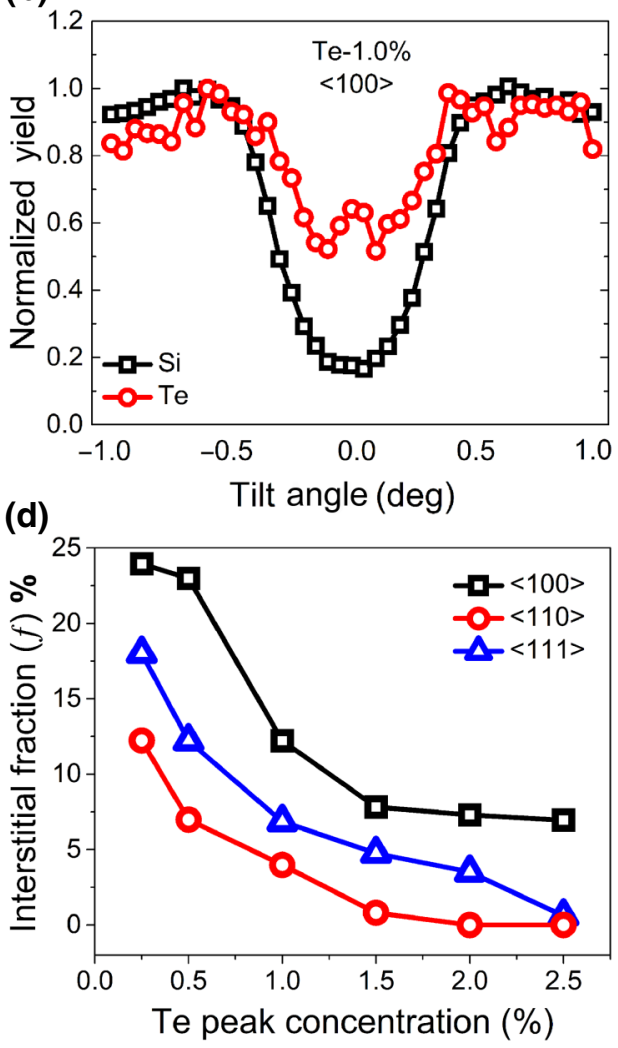

FIG. 2. (a),(b),(c) Angular distributions about the $\langle 100\rangle$ axes for $1.7 \mathrm{MeV} \mathrm{He}^{+}$scattered from $\mathrm{Te}$ (red circles) and Si (black squares) for the selected samples with different $\mathrm{Te}$ concentrations. The angular scan of substitutional Te will overlap with that of $\mathrm{Si}$ as reported. A peak is expected to arise in the middle of the angular scan for interstitial Te ions. The angular scan of substitutional but slightly displaced Te atoms is expected to be narrow and shallow. (d) The interstitial fraction of $\mathrm{Te}$ as a function of the concentration as calculated from the angular scan results.
[19,20,36-43], the electron concentration increases monotonically as the doping concentration increases and does not show any sign of saturation even at an atomic dopant concentration as high as $2.5 \%$ (exceeding $10^{21} \mathrm{~cm}^{-3}$ ). The unsaturated carrier concentration in the Te-hyperdoped $\mathrm{Si}$ layers is approaching $10^{21} \mathrm{~cm}^{-3}$, which is the value required for the next generation of Si technology $[1,4,44]$. This shows an outstanding behavior of deep impurities as compared with traditional $n$-type dopants in $\mathrm{Si}$ (e.g., As and $\mathrm{Sb}$ ) at very high doping levels, where the reported carrier concentration is never found to be larger than $6.5 \times 10^{20} \mathrm{~cm}^{-3}$ [45-47]. Figure 1(b) shows the room-temperature resistivity of Te-hyperdoped layers as the function of dopant concentration. Previous results for group-V dopants (i.e., As, P) doped Si [36,38-40,48] are also included. The smallest room-temperature resistivity of Te-hyperdoped Si samples is as low as $7.5 \times 10^{-4} \Omega \mathrm{cm}$ and is comparable to the smallest resistivity value for Asdoped $\mathrm{Si}$. In addition, the carrier mobility has also been obtained from the Hall effect measurements. As shown in Fig. 1(c), the mobility is in the range of $10-40 \mathrm{~cm}^{2} / \mathrm{V} \mathrm{s}$, which lies in the same order of the reported values for Asdoped $\mathrm{Si}$ at a high doping regime $\left(10^{20-21} \mathrm{~cm}^{-3}\right)$ [39] (see Appendix $\mathrm{C}$ for further details about the electrical transport measurements). IMT is also observed in Te-hyperdoped samples as shown by their temperature-dependent conductivity (see Fig. 5 in Appendix C). Samples Te- $0.25 \%$ and $\mathrm{Te}-0.50 \%$ with donor concentrations below the Mott limit (see Appendix C) show an insulating behavior with a strong temperature-dependent conductivity. However, samples with the Te peak concentration exceeding $1.0 \%$ present a radically different behavior, exhibiting a conductivity with negligible variation with temperature and a finite value of more than $10^{2} \mathrm{~S} / \mathrm{cm}$ as the temperature tends to zero. It is therefore crucial to understand this anomalous dependence of the electrical activation on the dopant concentration in Te-hyperdoped Si by scrutinizing the dopant lattice configuration at the atomic scale.

\section{B. Lattice location of Te dopants in Si matrix}

RBS-C is a unique technique to measure the lattice location of the dopants [49] (see Appendix D for further details). Figure 2 shows the angular distributions of $\mathrm{He}$ ions backscattered by $\mathrm{Te}$ and $\mathrm{Si}$ as a function of the tilt angle around the $\mathrm{Si}\langle 100\rangle$ axes in the selected samples. More angular scans in the vicinity of the three main crystallographic directions $(\langle 100\rangle,\langle 110\rangle$, and $\langle 111\rangle)$ are displayed in Appendix E. Two typical parameters are used to characterize the angular distributions, $\chi_{\min }$ and $\psi_{1 / 2}$. The minimum yield in the angular scan, $\chi_{\min }$, qualitatively describes the ability of dopants to block the channels. The critical angle $\psi_{1 / 2}$ represents the half width at half maximum of the angular scans between $\chi_{\min }$ and 1 . It is related 
to the displacement of substitutional atoms [50-52]. Both parameters are calculated for $\mathrm{Si}$ and $\mathrm{Te}$ at the same depth. The main results and their implications are as follows:

(i) For sample Te- $0.25 \%$, there is a peak in the middle of the angular scan. Some Te ions are located at low-symmetry interstitial sites lying around the axes of the crystal lattice. This peak decreases with increasing Te concentration. At low concentrations, Te ions occupy both substitutional and interstitial sites, whereas at higher concentration they preferably go to substitutional sites.

(ii) The interstitial fraction estimated from the angular scan drastically decreases at Te concentrations up to $1.0 \%$ and tends to a small constant value at higher concentrations [Fig. 2(d)]. It is worth noting that $1.0 \%$ is also the critical concentration where the IMT takes place in Te-doped Si [see Fig. 1(a)]. This result indicates a strong correlation between the atomic configuration of $\mathrm{Te}$ ions and the electronic properties of the samples.

(iii) At higher concentrations, $\psi_{1 / 2}$-Te is narrower compared to $\psi_{1 / 2}$-Si. This suggests that the Te dopants do not perfectly substitute the $\mathrm{Si}$ lattice sites. There exists a displacement $\left(r_{0}\right)$ from the ideal substitutional site [49]. Based on the experimental data, the projections of the displacement $r_{0}$ of Te atoms perpendicular to the three main axes can be estimated (see Appendix F). The average value $r$ obtained from the angular scan is found to be around $0.42 \pm 0.03 \AA$. This result is in agreement with the average displacement $\left(r_{0}=\right.$ $0.46 \AA$, for Te concentration of $1.36 \%$ ) obtained from the first-principles calculations shown later.

To further prove that the majority of Te ions are located at the substitutional lattice sites at high Te concentrations, we perform angular-scan mapping around three major crystalline axes. Figure 3 shows representative angulardependent two-dimensional backscattering-yield patterns of the sample with the highest Te concentration (Te-2.5\%). The backscattering yield patterns are extracted from the integral backscattering signals of $\mathrm{Si}$ and $\mathrm{Te}$ in the vicinity of $\langle 100\rangle,\langle 110\rangle$, and $\langle 111\rangle$ in sample Te-2.5\%. Prominent channeling effects are observed for both the Si and the Te signals and their patterns show a high similarity along $\langle 100\rangle,\langle 110\rangle$, and $\langle 111\rangle$ orientations and the closestpacked planes $(\{100\},\{110\}$, and $\{111\})$. These angularscan mappings unambiguously demonstrate that the Tehyperdoped Si layer is epitaxially regrown during the PLM treatment and that the majority of Te atoms occupy the substitutional sites of the Si matrix at such high Te concentrations.

\section{Ab initio calculations}

We directly probe the lattice location of $\mathrm{Te}$ ions in hyperdoped Si by RBS-C. These results suggest that the decrease of Te interstitials is accompanied by an increase of displacements of Te atoms from the ideal substitutional site. To understand this, first-principles calculations (see Appendix B for further computational details) have been carried out to compute the formation energy for three types of defects as a function of Te doping concentration. We define the formation energy of a defect $(D)$ as

$$
\Delta E_{F} \equiv E_{D}^{\mathrm{SC}}-n_{\mathrm{Si}}^{\mathrm{SC}} \mu_{\mathrm{Si}}-n_{\mathrm{Te}}^{\mathrm{SC}} \mu_{\mathrm{Te}},
$$

where $E_{D}^{\mathrm{SC}}$ is the total energy of the supercell (SC) with defect $D, n_{\mathrm{Si}}^{\mathrm{SC}}$ and $n_{\mathrm{Se}}^{\mathrm{SC}}$ are the numbers of $\mathrm{Si}$ and $\mathrm{Te}$ atoms in the supercell, and the chemical potentials $\mu_{\mathrm{Si}}$ and $\mu_{\mathrm{Te}}$ correspond to pure $\mathrm{Si}$ and $\mathrm{SiTe}_{2}$ in equilibrium with each other, respectively. In Fig. 4(a), we summarize our results for the most relevant types of defects: substitutional single $\mathrm{Te}\left(\mathrm{Te}_{\mathrm{Si}}\right)$ and substitutional Te dimer $\left(\mathrm{Te}_{\mathrm{Si}}-\mathrm{Te}_{\mathrm{Si}}\right)$. Moreover, the results for interstitial defects (Te interstitial at hexagonal $H$ and tetrahedral $T$ symmetry sites) at the selected concentrations are displayed in the inset of Fig. 4(a) for comparison. Generally, the formation energy of the interstitial sites ( $T$ site and $H$ site) is much larger (several $\mathrm{eV}$ per Te atom) than that of the substitutional site in the Te-hyperdoped Si system, which results in an energetically unfavorable formation of interstitial Te. Surprisingly, the $\mathrm{Te}_{\mathrm{Si}}-\mathrm{Te}_{\mathrm{Si}}$ dimer presents the lowest formation energy among all types of defects considered, and the difference in the formation energy (per Te atom) between a single $\mathrm{Te}_{\mathrm{Si}}$ dopant and a $\mathrm{Te}_{\mathrm{Si}}-\mathrm{Te}_{\mathrm{Si}}$ dimer is around $0.3 \mathrm{eV}$. This result suggests that $\mathrm{Te}_{\mathrm{Si}}-\mathrm{Te}_{\mathrm{Si}}$ dimers are more energetically favorable. Note that, at low concentrations, the average distance between Te atoms might be too large to promote the formation of dimers. As shown in Fig. 4(a), the $\mathrm{Te}_{\mathrm{Si}}$ formation energy increases sharply with increasing Te concentration on the insulating side, which is consistent with the previously reported results about Se-hyperdoped Si [22]. However, in the regime exceeding the threshold for the IMT, the defect formation energy is relatively stable; it only slightly increases with the Te concentration. Therefore, as the doping Te concentration increases, the penalty for assigning additional Te atoms becomes less costly and ultimately renders the $\mathrm{Te}_{\mathrm{Si}}-\mathrm{Te}_{\mathrm{Si}}$ dimer configuration stable. If $\mathrm{Te}_{\mathrm{Si}^{-}}-\mathrm{Te}_{\mathrm{Si}}$ dimers form, the lattice positions of Te are slightly displaced from the ideal substitutional sites. From the $a b$ initio relaxed positions of Te dimers, the displacement is estimated to be around $0.46 \AA$ for samples with a Te concentration of $1.36 \%$ (see Appendix F). The $\mathrm{Te}_{\mathrm{Si}}-\mathrm{Te}_{\mathrm{Si}}$ dimer formation and the displacement are in excellent agreement with the findings obtained from the experimental results shown in Figs. 2 and 3.

In Figs. 4(b) and 4(c) we show the density of states (DOS) corresponding to our simulations of $\mathrm{Te}_{\mathrm{Si}}-\mathrm{Te}_{\mathrm{Si}}$ and single $\mathrm{Te}_{\mathrm{Si}}$ at a $\mathrm{Te}$ concentration of $1.56 \%$, i.e., in the metallic regime. The projected DOS on the Te atomic 


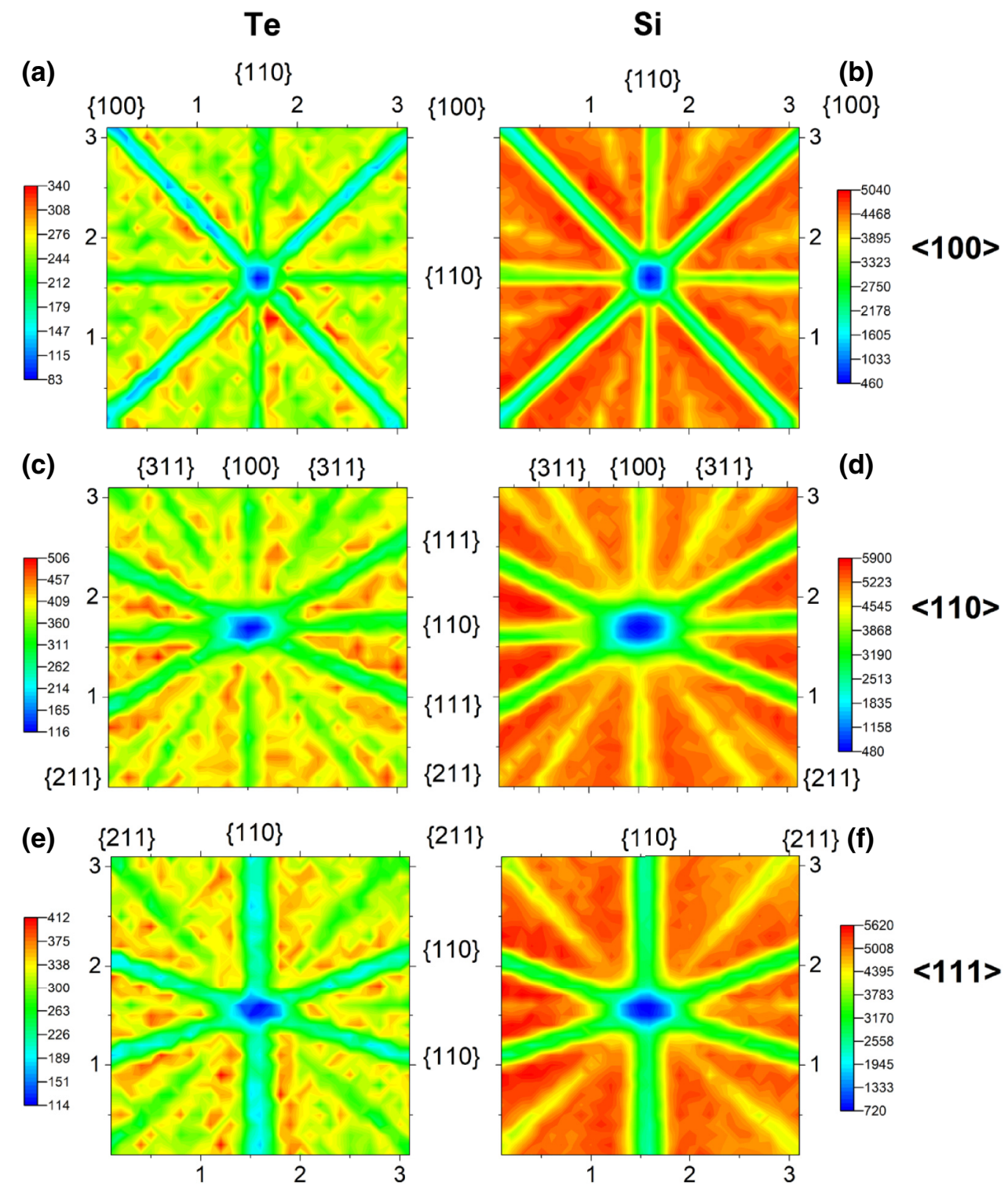

FIG. 3. Experimental twodimensional backscattered-yield patterns of the sample Te-2.5\% obtained from RBS-C spectra. The patterns are extracted from the integral backscattering signal from $\mathrm{Te}[(\mathrm{a}),(\mathrm{c})$, and (e)] and $\mathrm{Si}$ $[(\mathrm{b}),(\mathrm{d})$, and (f)] in the vicinity of the $\langle 100\rangle,\langle 110\rangle$, and $\langle 111\rangle$ directions. The patterns for the $\mathrm{Si}$ and the Te signals match well with each other, indicating that the majority of Te impurities are substitutional.

$<110>$

orbital has been summed up and displayed for Te single (red solid line) and Te dimer (black solid line); the Te projected DOS represents the contribution of electronic states localized at Te atoms to the total DOS. Figures 4(b) and 4(c) show a small gap approximately centered at $-0.7 \mathrm{eV}$ for $\mathrm{Te}_{\mathrm{Si}}$ and $-0.4 \mathrm{eV}$ for $\mathrm{Te}_{\mathrm{Si}}-\mathrm{Te}_{\mathrm{Si}}$, respectively, that separates the conduction band from the valence band. The modification of the bulk Si band gap is caused by doping. The Fermi energy (the zero of the horizontal energy scale) is located in the conduction band. By comparing the contribution of projected DOS on Te species with the total DOS, we notice that for the conduction band the Te projected DOS is small, denoting that the conduction electrons are delocalized on the whole crystal, thus producing a metallic phase. By performing the same comparison for the valence band, we can notice that for the dimer case the gap between the valence and conduction band is significantly reduced with respect to the single Te case, because of the presence of electronic states (of $s$ and $p$ character) at about $-0.7 \mathrm{eV}$ that are localized at the Te atoms, as can be seen in Fig. 4(c) by noticing the significant contribution of Te projected DOS to the total DOS at this energy. In both panels, the position of the Fermi energy denotes a metallic phase in agreement with the experimental data in Fig. 1(a). By integration of the local DOS from the conduction band minimum (CBM) up to the Fermi energy, we compute the number of carriers provided by each type of defect. From our ab initio simulations, we find that a single $\mathrm{Te}_{\mathrm{Si}}$ acts as a double donor, which is consistent with other reports $[53,54]$. At variance, one $\mathrm{Te}_{\mathrm{Si}}-\mathrm{Te}_{\mathrm{Si}}$ dimer provides only two electrons to the conduction band; i.e., each Te composing the dimer provides only one electron to the conduction band. From Fig. 1 we can notice that at high Te concentration, the electron concentration 

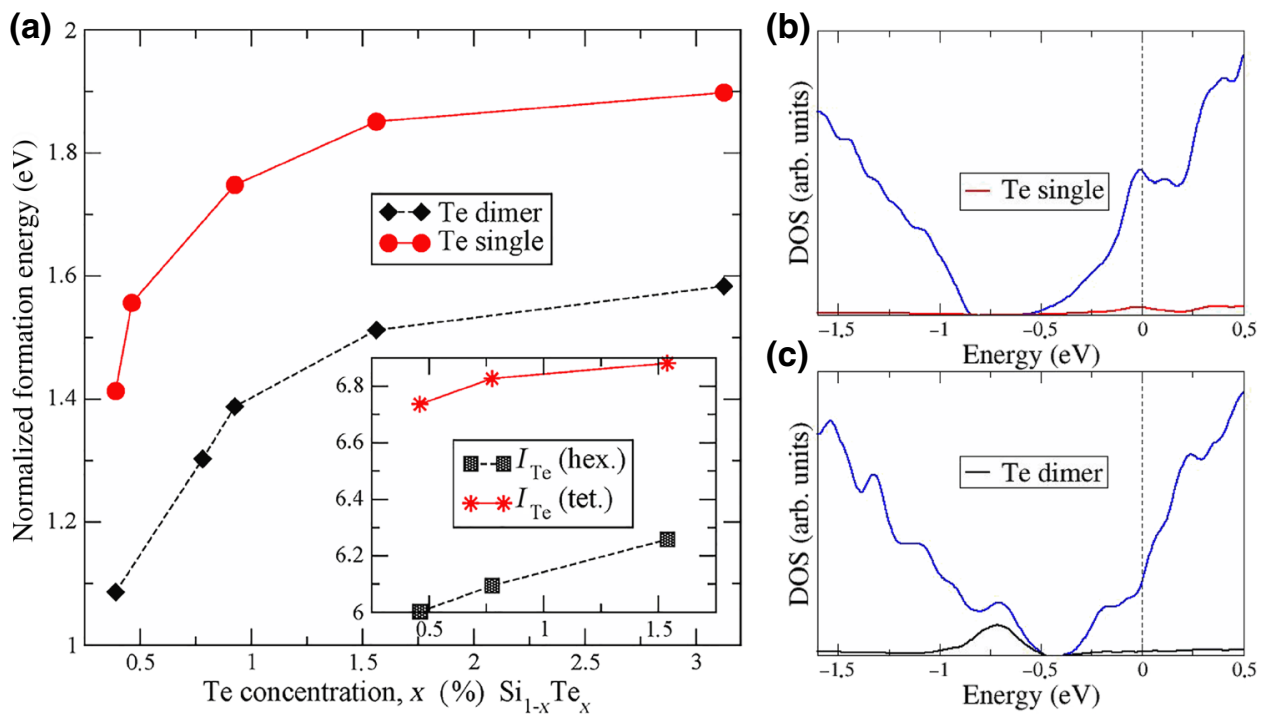

FIG. 4. (a) Normalized (per atom) formation energy $\left(\Delta E_{F} / n_{\mathrm{Te}}^{\mathrm{SC}}\right)$ of an isolated substitutional Te (circles) and a Te-Te dimer (diamonds) as a function of Te concentration, computed via $a b$ initio calculations. Inset: Formation energy of high-symmetry interstitial sites ( $T$ and $H$ denoted with squares and stars, respectively) as a function of Te concentration; lines are a guide for the eye. Ab initio calculations of density of states (DOS) at a Te concentration $x=1.56 \%$ for a single Te dopant (b) and a $\mathrm{Te}_{\mathrm{Si}}-\mathrm{Te}_{\mathrm{Si}}$ dimer (c). The sum of the DOS projections on the Te atomic orbitals is also displayed. The zero of the energy scales (dashed lines) corresponds to the Fermi energy.

$\left(8.1 \times 10^{20} \mathrm{~cm}^{-3}\right)$ is approaching the dopant concentration $\left(1.25 \times 10^{21} \mathrm{~cm}^{-3}\right)$ due to the formation of $\mathrm{Te}_{\mathrm{Si}}-\mathrm{Te}_{\mathrm{Si}}$ dimers. The lowest resistivity in our films is also comparable with As- and P-doped Si for similar doping levels [as shown in Fig. 1(b)]. We anticipate that the electron concentration can be further increased by optimizing the Te implantation fluence and annealing parameters, therefore opening a new avenue toward ultrahigh $n$-type doping for the Si-based new-generation microelectronics. As a final remark, we cannot exclude the appearance of $\mathrm{Te}_{n} V$ complexes from RBS-C $[55,56]$. However, the measured high carrier concentration supports the idea that $\mathrm{Te}_{n} V$ complexes cannot be the major states since they are electrically inactive centers $[7,12,14,16,20]$.

\section{CONCLUSION}

In conclusion, we demonstrate that the long-standing $n$-type doping limit in Si can be overcome by using deeplevel donors (e.g., Te) instead of traditional shallow-level donors (e.g., P, As, Sb). We have provided direct experimental and theoretical evidence for the formation of $\mathrm{Te}_{\mathrm{Si}^{-}}$ $\mathrm{Te}_{\mathrm{Si}}$ dimers with increasing Te concentration that are found to be responsible for the nonsaturating electron concentrations and the insulator-to-metal transition. This work provides insight toward understanding the lattice configurations and electronic structures of deep-level dopants in semiconductors.

\section{ACKNOWLEDGMENTS}

The authors thank Wolfhard Möller for fruitful discussion. Support by the Ion Beam Center (IBC) at HZDR is gratefully acknowledged. This work is funded by the Helmholtz-Gemeinschaft Deutscher Forschungszentren (HGF-VH-NG-713). M.W. acknowledges financial support by Chinese Scholarship Council (File No. 201506240060). A.D. acknowledges CINECA for computational resources allocated under the ISCRA initiative (IMeTe project) and thanks R. Colnaghi for technical support on computer hardware. Y.B. would like to thank the Alexander-von-Humboldt foundation for providing a postdoctoral fellowship.

\section{APPENDIX A: EXPERIMENTAL DETAILS}

The single-side polished Si (100) substrates (intrinsic, $\rho \geq 10^{4} \Omega \mathrm{cm}$ ) are implanted with Te ions at six different fluences at room temperature with implantation energies of 150 and $50 \mathrm{keV}$ (see Table I). The combined implantation energies are used to obtain a uniform distribution of $\mathrm{Te}$ in the implanted layer. The peak Te concentrations $\left(c_{p k}\right)$ are firstly calculated using SRIM code [57] and then verified by RBS-C measurements and SIMNRA $[58,59]$ simulation. The ion-implanted samples are exposed to a single laser pulse with the energy density of $1.2 \mathrm{~J} / \mathrm{cm}^{2}$. The whole amorphous implanted region is melted and then recrystallized with a solidification speed of the order of $10 \mathrm{~m} / \mathrm{s}$ while cooling down [60] during the annealing process. After 
TABLE I. Sample definition and related parameters. The sample names refer to the peak Te concentration of the as-implanted layer.

\begin{tabular}{|c|c|c|c|}
\hline \multirow[b]{2}{*}{ Sample name } & \multicolumn{2}{|c|}{ Implantation parameters } & \multirow{2}{*}{$\begin{array}{c}\text { Tellurium peak } \\
\text { concentration }\left(\mathrm{cm}^{-3}\right)\end{array}$} \\
\hline & Implantation energy $(\mathrm{keV})$ & Implantation doses $\left(\mathrm{cm}^{-2}\right)$ & \\
\hline \multirow[t]{2}{*}{ Te- $0.25 \%$} & 150 & $7.8 \times 10^{14}$ & $1.25 \times 10^{20}$ \\
\hline & 50 & $3.1 \times 10^{14}$ & \\
\hline \multirow[t]{2}{*}{$\mathrm{Te}-0.50 \%$} & 150 & $1.6 \times 10^{15}$ & $2.50 \times 10^{20}$ \\
\hline & 50 & $6.2 \times 10^{14}$ & \\
\hline \multirow[t]{2}{*}{$\mathrm{Te}-1.0 \%$} & 150 & $3.1 \times 10^{15}$ & $5.00 \times 10^{20}$ \\
\hline & 50 & $1.2 \times 10^{15}$ & \\
\hline \multirow[t]{2}{*}{$\mathrm{Te}-1.5 \%$} & 150 & $4.7 \times 10^{15}$ & $7.50 \times 10^{20}$ \\
\hline & 50 & $1.9 \times 10^{15}$ & \\
\hline \multirow[t]{2}{*}{$\mathrm{Te}-2.0 \%$} & 150 & $6.2 \times 10^{15}$ & $1.00 \times 10^{21}$ \\
\hline & 50 & $2.5 \times 10^{15}$ & \\
\hline \multirow[t]{2}{*}{$\mathrm{Te}-2.5 \%$} & 150 & $7.8 \times 10^{15}$ & $1.25 \times 10^{21}$ \\
\hline & 50 & $3.1 \times 10^{15}$ & \\
\hline
\end{tabular}

that, the Te-rich layer is recrystallized and its thickness is approximately $120 \mathrm{~nm}$ [33].

The RBS-C measurements are carried out using a 1-mmdiameter collimated 1.7-MeV He${ }^{+}$beam of the Rossendorf van de Graaff accelerator with a 10-20-nA beam current. Energy spectra of backscattered He are detected at an angle of $170^{\circ}$ with respect to the incoming beam direction using silicon surface barrier detectors with an energy resolution of $15 \mathrm{keV}$. Because of the so-called channeling effect, the backscattering probability sensitively depends on the direction of the incident beam with respect to the crystallographic orientation of the sample and is significantly reduced around the crystal axes and planes [61]. Angular axial scans are performed using a two-axis goniometer by recording integral backscattering yields in energy windows corresponding to scattering from $\mathrm{Si}$ and $\mathrm{Te}$ atoms (see Fig. 10) as a function of the angle of incidence around different crystallographic axial directions $(\langle 100\rangle,\langle 110\rangle$, and $\langle 111\rangle)$. In addition, two-dimensional angular backscattering patterns are obtained.

The electrical properties of the Te-hyperdoped Si samples are examined using a commercial Lake Shore Hall system in van der Pauw geometry [34]. Samples are measured in the temperature range from 2 to $300 \mathrm{~K}$ and a magnetic field perpendicular to the sample plane is swept from -6 to $6 \mathrm{~T}$. The gold electrodes are sputtered onto the four corners of the squarelike samples. The native $\mathrm{SiO}_{2}$ layer is removed by hydrofluoric acid (HF) etching prior to the sputtering process. Silver glue is used to contact the wires to the gold electrodes.

\section{APPENDIX B: COMPUTATIONAL DETAILS}

We performstructural and electronic calculations in the framework of the DFT, solving the Kohn-Sham equations through the plane-wave pseudopotential approach, as implemented in the QE open-source code [62]. We use ultrasoft pseudopotentials [63-66] in the separable form introduced by Kleinmann and Bylander [67], generated with a Perdew-Burke-Ernzerhof (PBE) exchangecorrelation functional and smearing techniques with a Gaussian broadening of $5 \mathrm{mRy}$. We choose a 50-Ry cutoff radius for the electronic valence wave function and 500-Ry cutoff radius for the charge density. The formation energy is computed according to Eq. (1) of the main text; the more natural choice for $\mu_{\mathrm{Si}}$ corresponds to the chemical potential of bulk silicon (usually referred as the Si-rich chemical potential), evaluated according to the standard procedure (see, e.g., Refs. [68,69]) by taking the total energy of the unit cell computed by DFT divided by the number of atoms contained in the unit cell. Our choice for $\mu_{\mathrm{Se}}$ corresponds to the Te chemical potential of bulk $\mathrm{SiTe}_{2}$ [18] in equilibrium with bulk silicon. So, $\mu_{\mathrm{Se}}$ is evaluated by subtracting the chemical potential of bulk Si from the total energy of the $\mathrm{SiTe}_{2}$ unit cell computed by DFT and dividing the result by 2 . Note that, for the present case, the choice of the chemical potential of Te simply shifts the zero of the energy, i.e., the vertical axis in Fig. 4(a) in the main text.

The $a b$ initio simulations of doped silicon are performed by using the supercell method. In a similar way as done, e.g., in Ref. [22], we vary the size of the supercell to simulate different concentrations of impurities (in our case, the Te concentration ranges from approximately $0.4 \%$ to $3.1 \%$ ). Periodic (i.e., Born-von Karman) boundary conditions are used. So, in the hyperdoping regime considered in the present work, each impurity interacts with its own images, simulating a uniform distribution of the same defect type. This approximation is expected to provide qualitative estimation of average properties like the defect formation energy of defect types representing a fraction of the total defect population, while it is expected to provide a reliable quantitative estimation of 
average properties of defect types representing the large majority of defects present in the sample. In the case in which only one type of defect is present or the concentration of the other types of defects is negligible, the present method would provide exact results within the density functional approximation technique used. In general, the atomic positions and the lattice constants are fully relaxed by the Broyden-Fletcher-Goldfarb-Shanno (BFGS) algorithm [21] with a threshold on the forces of $1 \mathrm{mRy} / a_{0}$. However, since we find that lattice constants satisfy Vegard's law (i.e., the lattice parameter varies linearly as a function of doping concentration) also for the higher doping regime we consider, we fix the lattice parameter for a few structures at a diluted concentration (thus having the largest supercell size considered in the present simulations) by linear interpolation of structurally relaxed results and relax only the atomic positions by the BFGS algorithm.

\section{APPENDIX C: ELECTRICAL TRANSPORT MEASUREMENTS}

The Te-hyperdoped Si samples with the single-side polished intrinsic Si substrate $\left(\rho>10^{4} \Omega \mathrm{cm}\right)$ are used for the electrical characterization. The Hall measurements and resistivity measurements of samples are measured using the van der Pauw technique [34]. Based on the basic physical principle of the Hall effect, the carrier density of the samples can be estimated using standard techniques and a magnetic field $\mathrm{B}$ ranging from -4 to $4 \mathrm{~T}$. In some cases, it is convenient to use a layer or sheet density $\left(n_{s}\right)$ instead of bulk density by using $n_{s}=I B / e \gamma V_{H}$, where $I$ is the current, $B$ is the magnetic field, $V_{H}$ is the Hall voltage, $e\left(1.602 \times 10^{-19} \mathrm{C}\right)$ is the elementary charge, and $\gamma$ is the Hall scattering factor that is generally assumed to be 1 heavily doped $\mathrm{Si}$. If the conducting layer thickness $d$ is known, the bulk density $(N)$ can be determined as $N=n_{s} / d$ [34]. Here, the carrier concentration $(n)$ ranges from $2.0 \times 10^{19}$ to $8.3 \times 10^{20} \mathrm{~cm}^{-3}$, which is calculated by assuming the effective thickness of the tellurium-doped layer as $125 \mathrm{~nm}$ [33]. This relatively large magnitude of the donor concentration is comparable to the shallow-level impurity doped Si with just-metallic concentrations [70] and is consistent with the metalliclike samples in S- and Se-doped $\mathrm{Si}[21,22]$. Moreover, the carrier mobility can be obtained via $\mu=|V H| / I B R_{S}=1 / q n_{s} R_{S}$, where $R_{s}$ is the sheet resistance. The obtained carrier mobility is in the range of $10-40 \mathrm{~cm}^{2} / \mathrm{Vs}$ and decreases with increasing Te doping concentration. These values are almost of the same order as in Si heavily doped with As in the high doping regime $\left(10^{20-21} \mathrm{~cm}^{-3}\right)$ [39].

The sheet resistance $\left(R_{S}\right)$ of samples over the temperature range $2-300 \mathrm{~K}$ is measured. The bulk electrical resistivity $\rho$ is calculated using $\rho=R_{S} * d$ and the conductivity $\sigma=1 / \rho$. The effective layer depth $d$ is $125 \mathrm{~nm}$,

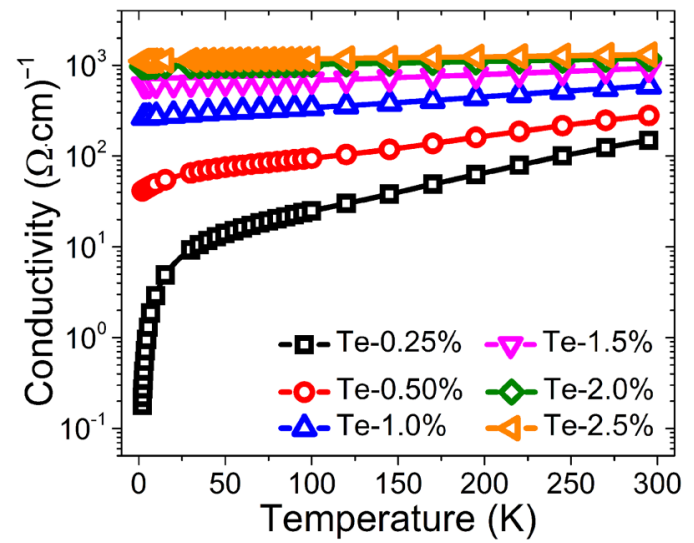

FIG. 5. The temperature-dependent conductivity of Tehyperdoped $\mathrm{Si}$ in the temperature range of 2-300 K. Samples with lower Te concentrations (Te- $0.25 \%$ and $\mathrm{Te}-0.50 \%)$ show a strong temperature-dependent conductivity, indicative of the insulating state. Samples with higher concentrations (Te-2.0\% and $\mathrm{Te}-2.5 \%$ ) exhibit a conductivity comparatively insensitive to temperature down to $2 \mathrm{~K}$, indicating an impurity-mediated transition to the metalliclike state.

which is verified by the RBS results (see Fig. 7). Figure 5 shows the temperature-dependent conductivity with the Te peak concentration varied from $1.25 \times 10^{20} \mathrm{~cm}^{-3}(0.25 \%)$ to $1.25 \times 10^{21} \mathrm{~cm}^{-3}(2.5 \%)$ in a temperature range of $2-300 \mathrm{~K}$. There, the room-temperature conductivity of Tehyperdoped $\mathrm{Si}$ samples is higher than $10^{2} \mathrm{~S} / \mathrm{cm}$, which confirms that the intrinsic Si substrate has no influence on the transport properties of the Te-hyperdoped Si layer considering the respective thickness. As shown in Fig. 5, a rigorous experimental evidence of an IMT lies in the measurement of nonzero conductivity as the temperature

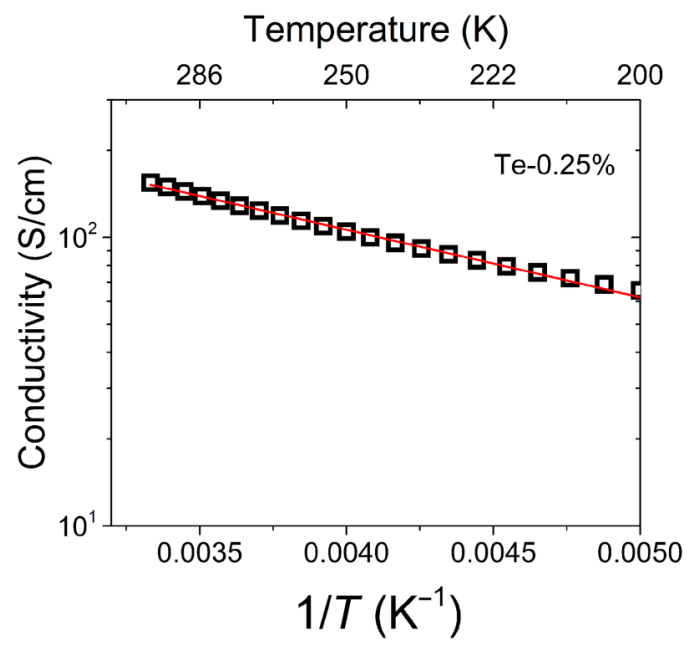

FIG. 6. The conductivity of sample Te- $0.25 \%$ as a function of inverse temperature. The red solid lines show the fits of data to Eq. (C3), yielding an activation energy of $46.5 \mathrm{meV}$. 
tends to zero (described previously [33]). Samples Te$0.25 \%$ and $\mathrm{Te}-0.50 \%$ show insulating behavior with a strong temperature-dependent conductivity. Samples with the Te peak concentration exceeding $1.0 \%$ present a dramatically different behavior, exhibiting a conductivity with negligible variation with temperature and a finite value of more than $10^{2} \mathrm{~S} / \mathrm{cm}$ as the temperature tends to zero. At $2 \mathrm{~K}$, the conductivity of the samples with $1.0 \%$ and $0.25 \%$ Te concentration differs by nearly 4 orders of magnitude in spite of the difference of just four times in the concentration. Samples with Te concentration exceeding $1.0 \%$ show a quasimetallic behavior. Moreover, the roomtemperature conductivity of Te-hyperdoped $\mathrm{Si}$ is around $600 \mathrm{~S} / \mathrm{cm}$, while the conductivity of S- and Se-hyperdoped $\mathrm{Si}$ is about 110 and $300 \mathrm{~S} / \mathrm{cm}$ respectively at similar doping levels (approximately $4.2-4.9 \times 10^{20} \mathrm{~cm}^{-3}$ ) [21,22].

As Mott [71,72] originally derived, the insulator-tometal transition in semiconductors can be described as

$$
n_{c}{ }^{1 / 3} a_{H}=0.25,
$$

where $a_{H}$ is the first Bohr radius of the donor electrons. The first Bohr radius can be scaled as

$$
a_{H}=\frac{e^{2}}{8 \pi \varepsilon_{0} \varepsilon_{r} E}
$$

where $E$ is the activation energy of the localized states, and $\epsilon_{0}$ and $\epsilon_{r}$ are the permittivity of free space and the highfrequency dielectric constant, respectively. Thus, by taking into account the activation energy as $199 \mathrm{meV}$ for a single Te atom [53,54] and $158 \mathrm{meV}$ [73] for a Te dimer and by assuming that the Mott criterion is approximately valid also for the dimer configuration, the isotropic Bohr radius is calculated as 3.1 and $3.9 \AA$, respectively. Therefore, the critical electron concentration for the Mott transition of a single $\mathrm{Te}$ atom in hyperdoped $\mathrm{Si}$ is approximately $5.24 \times 10^{20} \mathrm{~cm}^{-3}$, corresponding to a Te impurity concentration of $2.65 \times 10^{20} \mathrm{~cm}^{-3}$ since single substitutional Te atoms are double donors, while for a Te dimer in hyperdoped $\mathrm{Si}$, the critical electron concentration for the Mott transition is approximately $2.65 \times 10^{20} \mathrm{~cm}^{-3}$, corresponding to a Te impurity concentration of $2.65 \times 10^{20} \mathrm{~cm}^{-3}$ since one substitutional Te dimer donates two electrons. This rough estimation suggests that the onset for metallicity is almost the same for single Te or Te dimers.

Sample Te- $0.25 \%$ is away from the transition point. However, the Te atoms can be "ionized" even in sample $\mathrm{Te}-0.25 \%$ at room temperature because the large $\mathrm{Te}$ concentration gives rise to the formation of an impurity band that provides weakly localized or free electrons. To describe a thermally activated conduction process at high and low temperatures, we use

$$
\sigma=\frac{\sigma_{0}}{\exp \left[E_{0} / k_{B} T\right]},
$$

where the pre-exponential constant $\sigma_{0}$ and the activation energy $E_{0}$ are the free parameters. Over a limited temperature range, the measured conductivity data fit to this model quite nicely (as shown in Fig. 6). The fitting results indicate the thermally activated contribution to the conductivity of Te-hyperdoped Si. For sample Te- $0.25 \%$, a hightemperature activation region (300-200 K) with an activation energy of approximately $46.5 \mathrm{meV}\left(E_{0}\right)$ is observed, which is comparable to the thermal energy of room temperature (around $26 \mathrm{meV}$ ). Therefore, in sample Te- $0.25 \%$, it is possible to have a thermally activated conductivity. At low temperature, this sample shows variable-range hopping conductivity with a Coulomb gap. It can be fitted with a $1 / T^{1 / 2}$ dependence (not shown here).

\section{APPENDIX D: RBS-C MEASUREMENTS}

Figure 7 shows the representative RBS-C spectra of a PLM-treated Te-hyperdoped $\mathrm{Si}$ layer with different Te concentrations, also including the virgin $\mathrm{Si}$ and the as-implanted wafers. The random spectrum of the asimplanted layer reveals a thickness of about $120 \mathrm{~nm}$ for the Te profile, in an approximately Gaussian distribution. No channeling effect is observed in the implanted layer because of the amorphization caused by ion implantation. From the RBS-C signal, the PLM-treated layer shows a minimum backscattered yield $\chi_{\text {min }}$ of $4 \%$ (defined as the ratio of the aligned to the random yields near the sample surface from 740 to $920 \mathrm{keV}$ ), which is comparable to that of virgin Si substrate. This result indicates a full recrystallization and an epitaxial growth of the PLM-treated layers.

Moreover, a channeling behavior of Te (from 1380 to $1510 \mathrm{keV}$ ) is observed in the PLM-treated samples, which proves the incorporation of Te into the Si lattice sites. The

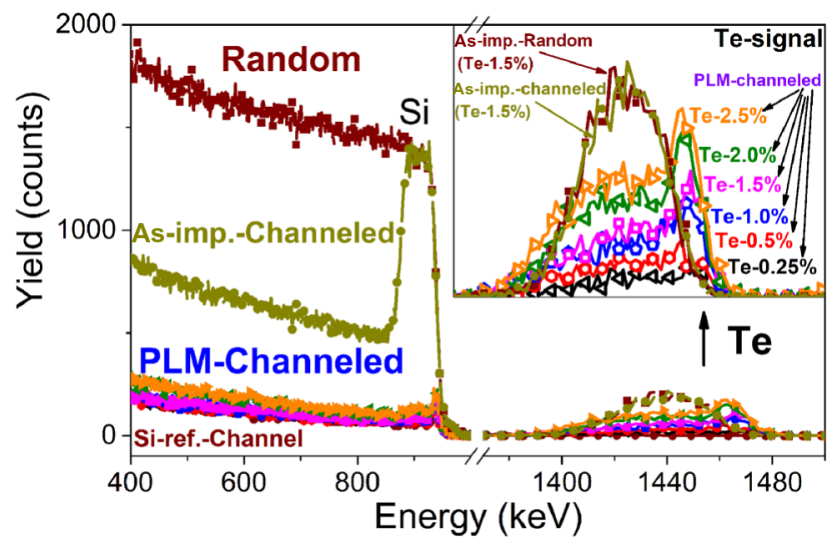

FIG. 7. A sequence of $1.7-\mathrm{MeV}$ He RBS-C spectra of asimplanted and PLM-treated Te-hyperdoped Si layers with different Te concentrations. The inset shows the magnification of random and channeling Te signals for the as-implanted and PLM-treated layers. 


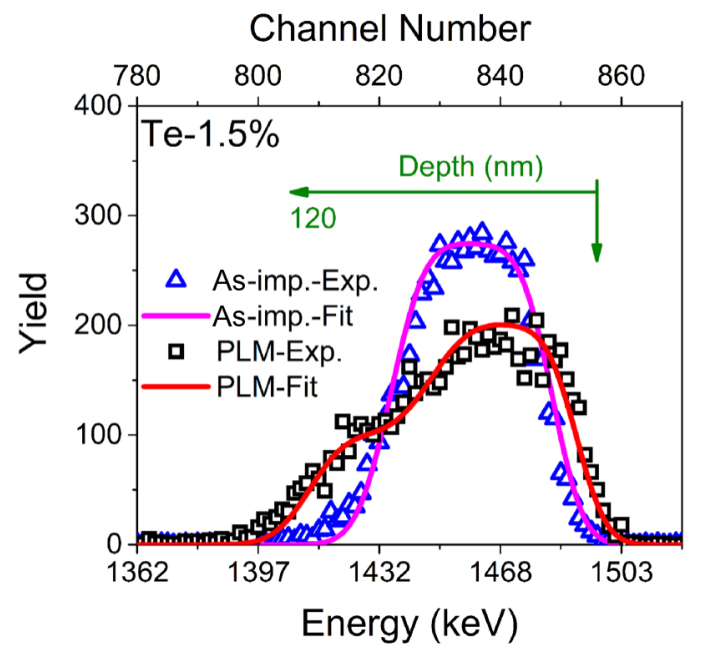

FIG. 8. The RBS random spectra and the fitting by the SIMNRA code for sample Te-1.5\% before and after the PLM treatment.

depth profile of the Te distribution before and after PLM treatment can be obtained by fitting RBS spectra using the SIMNRA code $[58,59]$. Figure 8 shows the Te signal taking from the random spectrum for sample Te-1.5\% before and after PLM treatment. It is evidenced in the Te signal that Te atoms tend to diffuse toward both the surface and the substrate side during the PLM process. The sample name is indicative of the peak Te concentration of the as-implanted layer. This has been verified in our previous work by transmission electron microscopy [33].

\section{APPENDIX E: DETAILED ANGULAR SCANS}

Figure 9(a) shows the schematic of the Si matrix with the implanted impurities that can take place at different lattice (I, II, III, and IV) sites. Figure 9(b) shows the angular scans for the respective lattice sites: the dashed line corresponds to the impurity, while the solid one to Si. If both angular scans match well with each other, impurities are located at the substitutional sites with high symmetry. The angular scan for impurities occupying interstitial sites is displayed in Fig. 9(b). Normally, two typical parameters are used to characterize the angular distributions, $\chi_{\min }$ and $\psi_{1 / 2}$. In detail, $\chi_{\min }$ corresponds to the minimum value of the backscattered ion angular spectrum with respect to the random direction normalized to 1 [52]. Qualitatively, it presents the ability of dopants blocking the channels. The critical angle $\psi_{1 / 2}$ represents the half width of the angular scans at half maximum between $\chi_{\min }$ and 1 (full width at half maximum of the angular scan spectrum), and it is related to the displacement vector of substitutional atoms [50].

Figure 10 shows the angular distributions for $\mathrm{Te}$ and $\mathrm{Si}$ at the same depth in the vicinity of the three main crystallographic directions, namely $\langle 100\rangle,\langle 110\rangle$, and $\langle 111\rangle$,

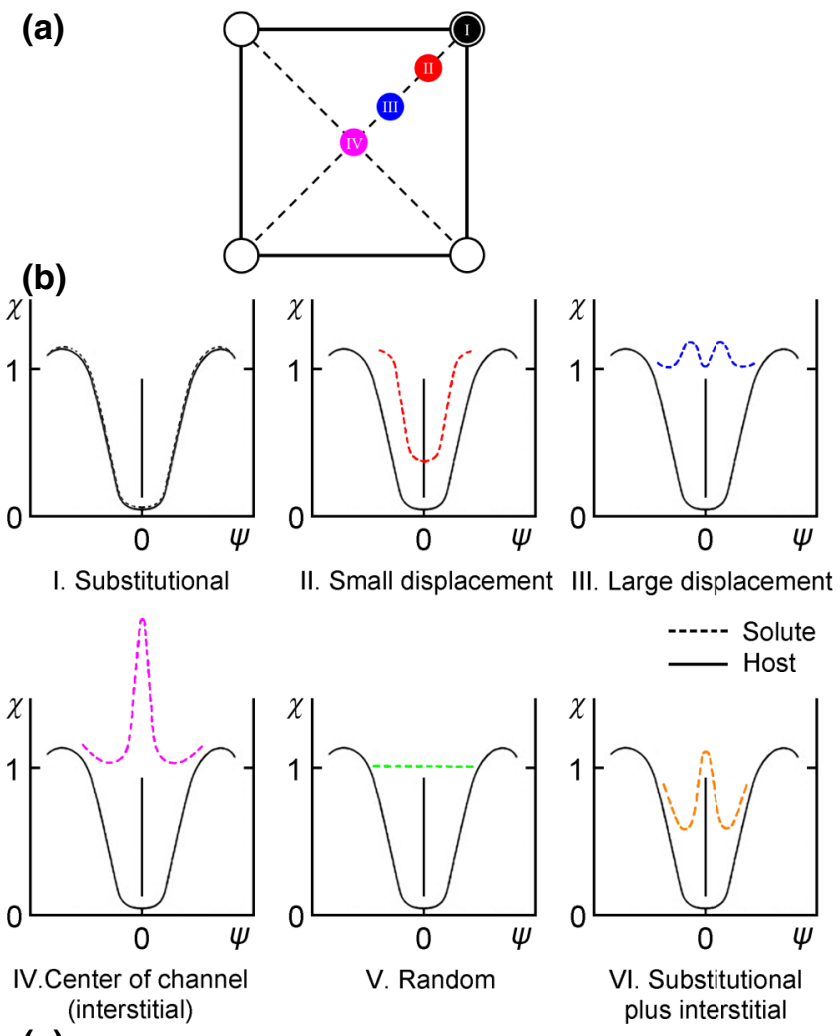

(c)

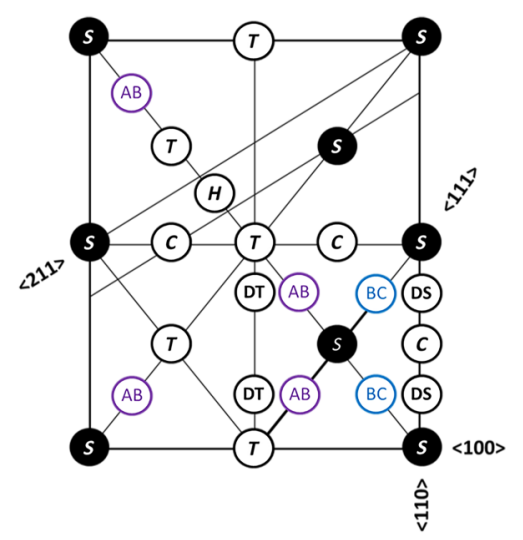

FIG. 9. The schematic structure of the Si matrix. (a) The solid black circle represents the impurity atom and the empty black circles stand for the host material atoms. The RBS-C angular scans for an impurity in the Si host material, at the substitutional site and interstitial site (channel center), are shown in (b) [50]. (c) Positions of major sites in the Si lattice [hexagonal $(\mathrm{H})$, tetrahedral (T), bond-centered (BC), antibonding (AB), split (SP), DS and DT sites (sites displaced from these high-symmetry sites)], shown in the 110 plane [74].

recorded off a major crystallographic plane. Two typical parameters $\chi_{\min }$ and $\psi_{1 / 2}$ are simultaneously calculated for $\mathrm{Si}$ and $\mathrm{Te}$ at the same depth. The three axial angular scans for Te follow those of the Si host in all the Te-hyperdoped Si samples, indicating that the dominant fraction of the Te atoms is located at substitutional $[50,75]$ sites. For samples $\mathrm{Te}-0.25 \%$ and $\mathrm{Te}-0.50 \%$, the $\chi_{\min }-\mathrm{Te}$ 

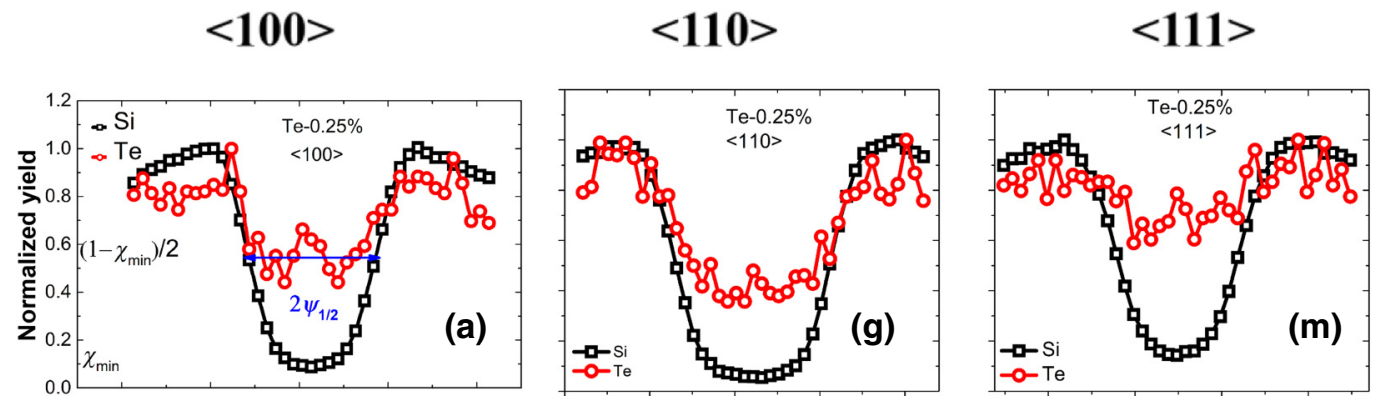

Te-0.25\%
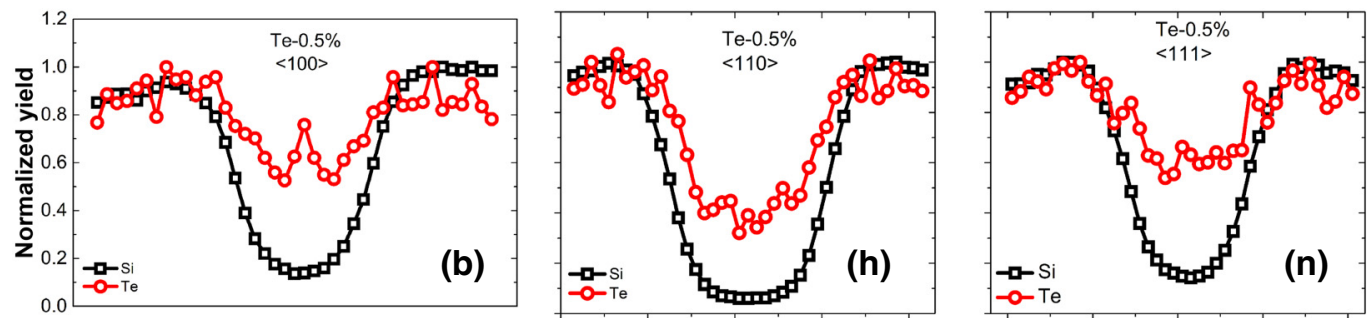

Te- $0.50 \%$
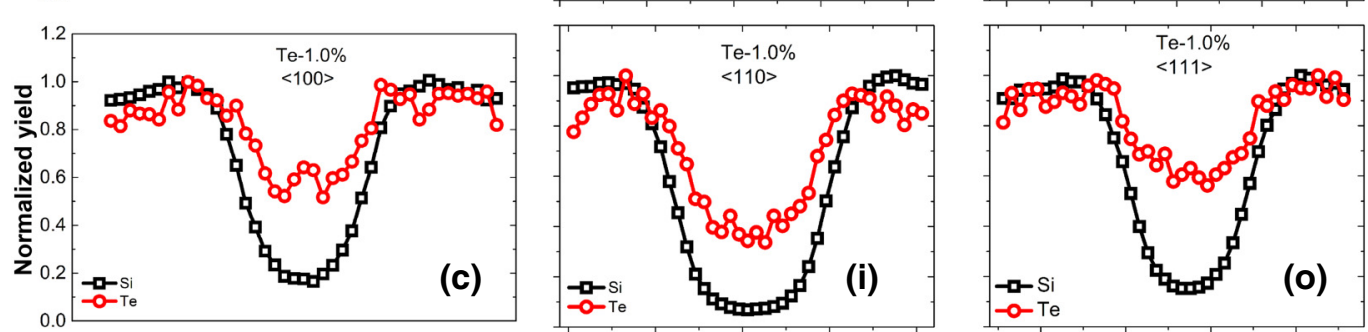

Te-1.0\%
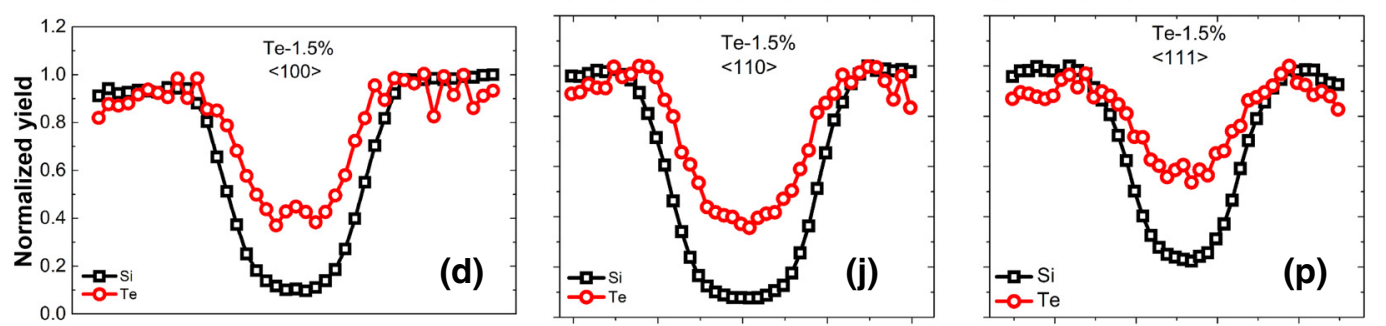

Te-1.5\%
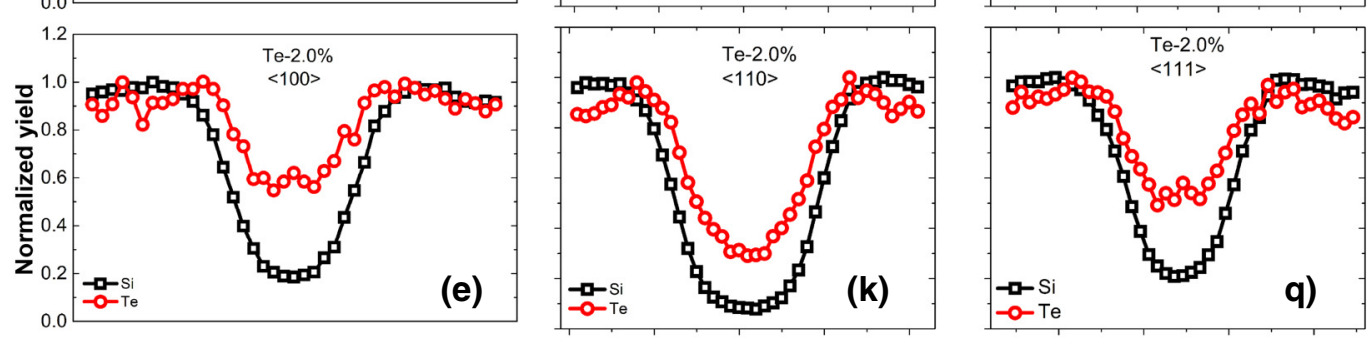

Te-2.0\%
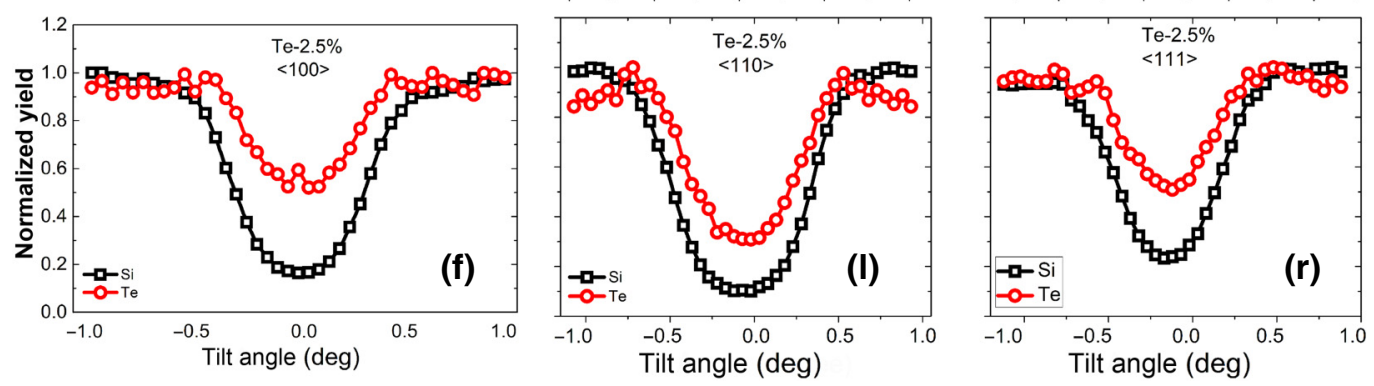

Te-2.5\%

FIG. 10. (a)-(r) The angular scans about the $\langle 100\rangle[(\mathrm{a})-(\mathrm{f})],\langle 110\rangle[(\mathrm{g})-(\mathrm{l})]$, and $\langle 111\rangle[(\mathrm{m})-(\mathrm{r})]$ axes for all the Te-hyperdoped Si samples. Angular distributions have been normalized in a random direction. The red circles correspond to Te atoms and black squares to $\mathrm{Si}$; smooth curves are drawn through the symbols. 
is significantly higher than that of $\mathrm{Si}$. This suggests that a non-negligible fraction of the Te atoms does not occupy substitutional sites and those atoms are located at the lowsymmetric interstitial sites lying around the axes of the crystal lattice. As depicted in Figs. 10(a), 10(b), 10(g), 10(h), 10(m), and 10(n), the interstitial site impurities are clearly evidenced. For samples with higher doping concentrations, a puzzling and interesting behavior is observed from the angular scans. The fraction of the interstitial Te atoms tends to vanish. As shown in Fig. 10 and contrary to the general expectation, the interstitial fraction decreases with increasing impurity concentration in Te-hyperdoped Si samples, which shows the similarity in the channeling direction of $\langle 100\rangle,\langle 110\rangle$, and $\langle 111\rangle$. The $\psi_{1 / 2}$-Te is narrower than that of $\psi_{1 / 2}$-Si (see Fig. 10) for all the Tehyperdoped Si samples in the three axial angular scans, meaning that the Te dopants do not perfectly substitute $\mathrm{Si}$ atoms from the host. There exists a displacement $\left(r_{0}\right)$ from the ideal $S$ site (i.e., $D S$ sites) [50]. In our case, the displacement is due to the formation of Te dimers. From the shape of the angular scan, we can state that single Te dopants dominate for the sample $0.25 \%$, while for samples with Te concentrations above $1.0 \%$, dimer Te dopants dominate, and the sample $0.50 \%$ is a mixed case. However, a quantitative estimation is difficult from the current data.

\section{APPENDIX F: TELLURIUM DISPLACEMENT ESTIMATION}

The RBS/C angular scans shown in Fig. 10 for $\mathrm{Si}$ and Te suggest that the Te atoms may be displaced from the ideal substitutional Si lattice sites. To obtain quantitative information about the Te location, the angular distributions as a function of the equilibrium displacement of an atom from a lattice row [51] are calculated. The parameter $\psi_{1 / 2}$ can be expressed as follows for displacement calculation [52]:

$$
\psi_{1 / 2}=\sqrt{\left[\frac{1}{2} \ln \left(\frac{C^{2} a^{2}}{r_{\min }^{2}}+1\right)\right]} \times \sqrt{\frac{2 Z_{1} Z_{2} e^{2}}{E d}},
$$

where constant $C^{2}$ is around $3 ; Z_{1}$ and $Z_{2}$ are the atomic numbers of the incident particle and of the considered atom, respectively; $e^{2}$ is equal to $14.4 \mathrm{eV} \AA ; E=1.7 \mathrm{MeV}$ is the incident ion energy, and $d$ is the average atomic distance in the considered atomic row $\left(d_{\mathrm{aver}}\langle 110\rangle=5.43 \AA\right.$ $d_{\text {aver }}\langle 110\rangle=3.84 \AA d_{\text {aver }}\langle 111\rangle=4.70 \AA$ ) [52]; and

$$
r_{\min }^{2}=\rho^{2} \ln 2+r_{0}^{2} .
$$

Here, $\rho$ is the thermal vibration amplitude and $r_{0}$ is the projection (on a plane perpendicular to the channeling direction) of the equilibrium displacement of the Te atoms with respect to the lattice sites. $a$ is the screening distance, which is related to the Bohr radius $\left(a_{0}=0.528 \AA\right)$,
TABLE II. The experimental values of the displacement are obtained from formula F1, which corresponds to the lattice sites projected perpendicularly to the channeling direction. The $\langle 100\rangle$ direction is perpendicular to the $\mathrm{Si}$ substrate. Moreover, the computational values of the displacement are from the firstprinciples calculations, where an average displacement of $0.46 \AA$

\begin{tabular}{|c|c|c|c|}
\hline \multicolumn{4}{|c|}{$r_{0}$ (experimental values) } \\
\hline & $r_{\langle 100\rangle}$ & $r_{\langle 110\rangle}$ & $r_{\langle 111\rangle}$ \\
\hline \multicolumn{4}{|l|}{ Te- $0.25 \%$} \\
\hline $\mathrm{Te}-0.50 \%$ & $0.36 \pm 0.03$ & $0.40 \pm 0.03$ & $0.34 \pm 0.03$ \\
\hline $\mathrm{Te}-1.0 \%$ & $0.37 \pm 0.03$ & $0.40 \pm 0.03$ & $0.36 \pm 0.03$ \\
\hline $\mathrm{Te}-1.5 \%$ & $0.42 \pm 0.03$ & $0.40 \pm 0.03$ & $0.38 \pm 0.03$ \\
\hline $\mathrm{Te}-2.0 \%$ & $0.43 \pm 0.03$ & $0.41 \pm 0.03$ & $0.45 \pm 0.03$ \\
\hline $\mathrm{Te}-2.5 \%$ & $0.46 \pm 0.03$ & $0.42 \pm 0.03$ & $0.46 \pm 0.03$ \\
\hline \multicolumn{4}{|c|}{$r_{0}$ (computational values) } \\
\hline & $r_{\langle 100\rangle}$ & $r_{\langle 110\rangle}$ & $r_{\langle 111\rangle}$ \\
\hline $\mathrm{Te}-1.36 \%$ & 0.38 & 0.37 & 0.33 \\
\hline
\end{tabular}
is obtained.

as follows:

$$
a=0.8853 \times a_{0} \times \frac{1}{\sqrt[3]{\left(Z_{1}^{1 / 2}+Z_{2}^{1 / 2}\right)^{2}}}=0.11114 .
$$

Here, the range for the average error of the projection displacement is determined by the dispersion of the parameter $\psi_{1 / 2}$.

For comparison, we computed the displacements obtained in our $a b$ initio simulations. To minimize the numerical error, which in our simulation is comparable to the variation of the displacement as a function of the Te concentration, we average the values of displacements of Te impurities obtained from first-principles calculations for $\mathrm{Te}_{\mathrm{Si}}-\mathrm{Te}_{\mathrm{Si}}$ over the whole range of the investigated concentration. The average results, corresponding to the average Te concentration of $1.36 \%$, are displayed in Table II (bottom panel) in good agreement with the experimental data for $1.0 \%$ and $1.5 \%$ Te concentrations the corresponding first principles average displacement is equal to $0.46 \AA$.

[1] International Technology Roadmap for Semiconductors (ITRS); Available at https://www.semiconductors.org/main/ 2015_international_technology_roadmap_for_semiconduct ors_itrs/.

[2] H.-J. Gossmann, C. S. Rafferty, and P. Keys, Junctions for deep sub-100 nm MOS: How far will ion implantation take us?, Mater. Res. Soc. Symp. 610, B1.2.1 (2000).

[3] A. Nylandsted Larsen, F. T. Pedersen, G. Weyer, R. Galloni, R. Rizzoli, and A. Armigliato, The nature of electrically inactive antimony in silicon, J. Appl. Phys. 59, 1908 (1986). 
[4] H-J Gossmann, F. C. Unterwald, and H. S. Luftman, Doping of Si thin films by low-temperature molecular beam epitaxy, J. Appl. Phys. 73, 8237 (1993).

[5] H. H. Radamson, M. R. Sardela Jr, L. Hultman, and G. V. Hansson, Characterization of highly Sb-doped Si using high-resolution x-ray diffraction and transmission electron microscopy, J. Appl. Phys. 76, 763 (1994).

[6] K. Saarinen, J. Nissila, H. Kauppinen, M. Hakala, M. J. Puska, P. Hautojarvi, and C. Corbel, Identification of Vacancy-Impurity Complexes in Highly -Type Si, Phys. Rev. Lett. 82, 1883 (1999).

[7] V. Ranki, K. Saarinen, J. Fage-Pedersen, J. Lundsgaard Hansen, and A. Nylandsted Larsen, Electrical deactivation by vacancy-impurity complexes in highly As-doped $\mathrm{Si}$, Phys. Rev. B 67, 041201 (2003).

[8] M. Rummukainen, I. Makkonen, V. Ranki, M. J. Puska, and K. Saarinen, Vacancy-Impurity Complexes in Highly SbDoped Si Grown by Molecular Beam Epitaxy, Phys. Rev. Lett. 94, 165501 (2005).

[9] M. Ramamoorthy and S. T. Pantelides, Complex Dynamical Phenomena in Heavily Arsenic Doped Silicon, Phys. Rev. Lett. 76, 4753 (1996).

[10] D. C. Mueller, E. Alonso, and W. Fichtner, Arsenic deactivation in Si: Electronic structure and charge states of vacancy-impurity clusters, Phys. Rev. B 68, 045208 (2003).

[11] A. Satta, E. Albertazzi, G. Lulli, and L. Colombo, Ab initio structures of AsmV complexes and the simulation of Rutherford backscattering channeling spectra in heavily As-doped crystalline silicon, Phys. Rev. B 72, 235206 (2005).

[12] V. Ranki, J. Nissilä, and K. Saarinen, Formation of Vacancy-Impurity Complexes by Kinetic Processes in Highly As-Doped Si, Phys. Rev. Lett. 88, 105506 (2002).

[13] D. Mathiot and J. C. Pfister, Diffusion of arsenic in silicon: Validity of the percolation model, Appl. Phys. Lett. 42, 1043 (1983).

[14] K. C. Pandey, A. Erbil, C. S. Cargill, R. F. Boehme, and D. Vanderbilt, Annealing of Heavily Arsenic Doped Silicon: Electrical Deactivation and a New Defect Complex, Phys. Rev. Lett. 61, 1282 (1988).

[15] D. Mathiot and J. C. Pfister, Dopant redistribution in heavily doped silicon: Confirmation of the validity of the vacancy-percolation model, J. Appl. Phys. 66, 970 (1989).

[16] D. W. Lawther, U. Myler, P. J. Simpson, P. M. Rousseau, P. B. Griffin, and J. D. Plummer, Vacancy generation resulting from electrical deactivation of arsenic, Appl. Phys. Lett. 67, 3575 (1995).

[17] R. Pinacho, M. Jaraiz, P. Castrillo, I. Martin-Bragado, J. E. Rubio, and J. Barbolla, Modeling arsenic deactivation through arsenic-vacancy clusters using an atomistic kinetic Monte Carlo approach, Appl. Phys. Lett. 86, 252103 (2005).

[18] D. Christoph Mueller and W. Fichtner, Highly n-doped silicon: Deactivating defects of donors, Phys. Rev. B 70, 245207 (2004).

[19] D. J. Chadi, P. H. Citrin, C. H. Park, D. L. Adler, M. A. Marcus, and H.-J. Gossmann, Fermi-Level-Pinning Defects in Highly n-Doped Silicon, Phys. Rev. Lett. 79, 4834 (1997).
[20] P. M. Voyles, D. A. Muller, J. L. Grazul, P. H. Citrin, and H.-J. L. Gossmann, Atomic-scale imaging of individual dopant atoms and clusters in highly n-type bulk Si, Nature 416, 826 (2002).

[21] M. T. Winkler, D. Recht, M.-J. Sher, A. J. Said, E. Mazur, and M. J. Aziz, Insulator-to-Metal Transition in SulfurDoped Silicon, Phys. Rev. Lett. 106, 178701 (2011).

[22] E. Ertekin, M. T. Winkler, D. Recht, A. J. Said, M. J. Aziz, T. Buonassisi, and J. C. Grossman, Insulator-to-Metal Transition in Selenium-Hyperdoped Silicon: Observation and Origin, Phys. Rev. Lett. 108, 026401 (2012).

[23] S. Zhou, F. Liu, S. Prucnal, K. Gao, M. Khalid, C. Baehtz, M. Posselt, W. Skorupa, and M. Helm, Hyperdoping silicon with selenium: solid vs. liquid phase epitaxy, Sci. Rep. 5, 8329 (2015).

[24] F. Liu, S. Prucnal, Y. Berencén, Z. Zhang, Y. Yuan, Y. Liu, R. Heller, R. Böttger, L. Rebohle, W. Skorupa, M. Helm, and S. Zhou, Realizing the insulator-to-metal transition in Se-hyperdoped Si via non-equilibrium material processing, J. Phys. D: Appl. Phys. 50, 415102 (2017).

[25] W. K. Chu and B. J. Masters, in AIP Conference Proceedings, 50, 305 (1979).

[26] J. L. Allain, J. R. Regnard, A. Bourret, A. Parisini, A. Armigliato, G. Tourillon, and S. Pizzini, Extended $\mathrm{X}$-ray-absorption fine-structure study of the local atomic structure in $\mathrm{As}^{+}$heavily implanted silicon, Phys. Rev. B 46, 9434 (1992).

[27] K. M. Yu, W. Walukiewicz, T. Wojtowicz, I. Kuryliszyn, X. Liu, Y. Sasaki, and J. K. Furdyna, Effect of the location of Mn sites in ferromagnetic on its Curie temperature, Phys. Rev. B 65, 201303(R) (2002).

[28] K. M. Yu, W. Walukiewicz, T. Wojtowicz, W. L. Lim, X. Liu, U. Bindley, M. Dobrowolska, and J. K. Furdyna, Curie temperature limit in ferromagnetic $G a_{1-x} \mathrm{Mn}_{\mathrm{x}}$ As, Phys. Rev. B 68, 041308(R) (2003).

[29] U. Wahl, L. M. Amorim, V. Augustyns, A. Costa, E. DavidBosne, T. A. L. Lima, G. Lippertz, J. G. Correia, M. R. da Silva, M. J. Kappers, K. Temst, A. Vantomme, and L. M. C. Pereira, Lattice Location of $\mathrm{Mg}$ in GaN: A Fresh Look at Doping Limitations, Phys. Rev. Lett. 118, 09550 (2017).

[30] G. Miceli and A. Pasquarello, Self-compensation due to point defects in Mg-doped GaN, Phys. Rev. B 93, 165207 (2016).

[31] S. Hu, P. Han, S. Wang, X. Mao, X. Li, and L. Gao, Structural and optoelectronic properties of selenium-doped silicon formed using picosecond pulsed laser mixing, Physica Status Solidi (a) 209, 2521 (2012).

[32] X. Qiu, X. Yu, S. Yuan, Y. Gao, X. Liu, Y. Xu, and D. Yang, Trap assisted bulk silicon photodetector with high photoconductive gain, low noise, and fast response by $\mathrm{Ag}$ hyperdoping, Adv. Opt. Mater. 6, 1700638 (2018).

[33] M. Wang, Y. Berencén, E. García-Hemme, S. Prucnal, R. Hübner, Y. Yuan, C. Xu, L. Rebohle, R. Böttger, R. Heller, H. Schneider, W. Skorupa, M. Helm, and S. Zhou, Extended Infrared Photoresponse in Te-Hyperdoped Si at Room Temperature, Phys. Rev. Appl. 10, 024054 (2018).

[34] L. Van der Pauw, A Method of Measuring the Resistivity and Hall Coefficient on Lamellae of Arbitrary Shape, Philips Tech. Rev. 20, 320 (1958). 
[35] P. Giannozzi, et al., QUANTUM ESPRESSO: a modular and open-source software project for quantum simulations of materials, J. Phys.: Condens. Matter 21, 395502 (2009).

[36] S. Solmi, M. Severi, R. Angelucci, L. Baldi, and R. Bilenchi, Electrical properties of thermally and laser annealed polycrystalline silicon films heavily doped with arsenic and phosphorus, J. Electrochem. Soc. 129, 1811 (1982).

[37] R. D. Chang and C. H. Lin, Activation and deactivation of phosphorus in silicon-on-insulator substrates, Mater. Sci. Semicond. Process. 42, 219 (2016).

[38] D. Giubertoni, G. Pepponi, M. A. Sahiner, S. P. Kelty, S. Gennaro, M. Bersani, M. Kah, K. J. Kirkby, R. Doherty, M. A. Foad, F. Meirer, C. Streli, J. C. Woicik, and P. Pianetta, Deactivation of submelt laser annealed arsenic ultrashallow junctions in silicon during subsequent thermal treatment, J. Vac. Sci. Technol. B 28, C1B1 (2010).

[39] G. Borot, L. Rubaldo, L. Clement, R. Pantel, D. Dutartre, K. Kuitunen, J. Slotte, F. Tuomisto, X. Mescot, M. Gri, and G. Ghibaudo, Tensile strain in arsenic heavily doped Si, J. Appl. Phys. 102, 103505 (2007).

[40] D. Giubertoni, G. Pepponi, S. Gennaro, M. Bersani, M. A. Sahiner, S. P. Kelty, R. Doherty, M. A. Foad, M. Kah, K. J. Kirkby, J. C. Woicik, and P. Pianetta, Correlation of local structure and electrical activation in arsenic ultrashallow junctions in silicon, J. Appl. Phys. 104, 103716 (2008).

[41] S. Solmi and D. Nobili, High concentration diffusivity and clustering of arsenic and phosphorus in silicon, J. Appl. Phys. 83, 2484 (1998).

[42] F. F. Komarov, O. I. Velichko, V. A. Dobrushkin, and A. M. Mironov, Mechanisms of arsenic clustering in silicon, Phys. Rev. B 74, 035205 (2006).

[43] C. W. White, P. P. Pronko, S. R. Wilson, B. R. Appleton, J. Narayan, and R. T. Young, Effects of pulsed ruby-laser annealing on As and Sb implanted silicon, J. Appl. Phys. 50, 3261 (1979).

[44] K. Suzuki, Y. Tada, Y. Kataoka, K. Kawamura, T. Nagayama, S. Nagayama, C. W. Magee, T. H. Buyuklimanli, D. C. Mueller, W. Fichtner, and C. Zechner, Maximum active concentration of ion-implanted phosphorus during solid-phase epitaxial recrystallization, IEEE Trans. Electron Device 54, 1985 (2007).

[45] Paul A. Packan, Pushing the limits, Science 285, 2079 (1999).

[46] A. Lietoila, J. F. Gibbons, and T. W. Sigmon, The solid solubility and thermal behavior of metastable concentrations of As in Si, Appl. Phys. Lett. 36, 765 (1980).

[47] S. Solmi, in Encyclopedia of Materials: Science and Technology, edited by K. H. J. Buschow, R. W. Cahn, M. C. Flemings, B. Ilschner, E. J. Kramer, S. Mahajan, and P. Veyssière (Elsevier Sci., New York, 2001), p. 2331.

[48] J. S. Williams and K. T. Short, Metastable doping behavior in antimony-implanted (100) silicon, J. Appl. Phys. 53, 8663 (1982).

[49] W.-K. Chu, Backscattering Spectrometry (Elsevier, New York, 2012).

[50] L. C. Feldman, J. W. Mayer, and S. T. Picraux, Materials Analysis by Ion Channeling: Submicron Crystallography (Academic Press, New York, 2012).
[51] S. T. Picraux, W. L. Brown, and W. M. Gibson, Lattice location by channeling angular distributions: $\mathrm{Bi}$ implanted in $\mathrm{Si}$, Phys. Rev. B 6, 1382 (1972).

[52] C. Brizard, J. R. Regnard, J. L. Allain, A. Bourret, M. Dubus, A. Armigliato, and A. Parisini, Backscattering spectrometry and ion channeling studies of heavily implanted $\mathrm{As}^{+}$in silicon, J. Appl. Phys. 75, 126 (1994).

[53] R. Schaub, G. Pensl, M. Schulz, and C. Holm, Donor states in tellurium-doped silicon, Appl. Phys. A 34, 215 (1984).

[54] K. Sánchez, I. Aguilera, P. Palacios, and P. Wahnón, Formation of a reliable intermediate band in Si heavily coimplanted with chalcogens ( $\mathrm{S}, \mathrm{Se}, \mathrm{Te})$ and group III elements (B, Al), Phys. Rev. B 82, 165201 (2010).

[55] S. Balboni, E. Albertazzi, M. Bianconi, and G. Lulli, Atomistic modeling of ion channeling in $\mathrm{Si}$ with point defects: The role of lattice relaxation, Phys. Rev. B 66, 045202 (2002).

[56] A. Satta, E. Albertazzi, M. Bianconi, G. Lulli, S. Balboni, and L. Colombo, Atomistic simulation of ion channeling in heavily doped Si: As, Nucl. Instrum. Methods B 230, 112 (2005).

[57] J. F. Ziegler, SRIM-2003, Nucl. Instrum. Methods Phys. Res. B 219, 1027 (2004).

[58] M. Mayer, SIMNRA user's guide, Report IPP 9/113 (Max-Planck-Institut für Plasmaphysik, Garching, Germany, 1997).

[59] M. Mayer, SIMNRA, a Simulation Program for the Analysis of NRA, RBS and ERDA (AIP Conf. Proc., 1999), pp. 541.

[60] F. Spaepen, D. Turnbull, J. Poate, and J. Mayer, Laser Annealing of Semiconductors (Academic, New York, 1982).

[61] W. Chu, J. Mayer, M. Nicolet, T. M. Buck, G. Amsel, and F. Eisen, Principles and applications of ion beam techniques for the analysis of solids and thin films, Thin Solid Films 17, 1 (1973).

[62] A. M. Rappe, K. M. Rabe, E. Kaxiras, and J. D. Joannopulos, Optimized pseudopotentials, Phys. Rev. B 41, 1227 (1990).

[63] D. Vanderbilt, Optimally smooth norm-conserving pseudopotentials, Phys. Rev. B 32, 8412 (1985).

[64] D. Vanderbilt, Soft self-consistent pseudopotentials in a generalized eigenvalue formalism, Phys. Rev. B 41, 7892R (1990).

[65] L. Kleinmann and D. M. Bylander, Efficacious Form for Model Pseudopotentials, Phys. Rev. Lett. 48, 1425 (1982).

[66] C. G. Broyden, The convergence of a class of double-rank minimization algorithms 1. general considerations, J. Appl. Math. 6, 76 (1970).

[67] B. K. Newman, E. Ertekin, J. T. Sullivan, M. T. Winkler, M. A. Marcus, S. C. Fakra, M.-J. Sher, E. Mazur, J. C. Grossman, and T. Buonassisi, Extended X-ray absorption fine structure spectroscopy of selenium-hyperdoped silicon, J. Appl. Phys. 114, 133507 (2013).

[68] G. Petretto, A. Debernardi, and M. Fanciulli, Confinement effects and hyperfine structure in Se doped silicon nanowires, Nano Lett. 11, 4509 (2011). 
[69] R. W. Wyckoff, Crystal Structures (Wiley Interscience Publication, New York, 1971), Vol. 1.

[70] P. Dai, Y. Zhang, and M. Sarachik, Electrical conductivity of metallic Si: B near the metal-insulator transition, Phys. Rev. B 45, 3984 (1992).

[71] N. F. Mott, Metal-insulator transition, Rev. Mod. Phys. 40, 677 (1968).

[72] N. F. Mott, Metal-insulator transitions, Contemp. Phys. 14, 401 (1973).

[73] P. Wagner, C. Holm, E. Sirtl, R. Oeder, and W. Zulehner, in Festkörperprobleme: Advances in Solid State Physics, edited by P. Grosse (Vieweg, Braunschweig, 1984), Vol. 24, p. 191.

[74] D. J. Silva, U. Wahl, J. G. Correia, L. M. C. Pereira, L. M. Amorim, M. R. da Silva, E. Bosne, and J. P. Araújo, Lattice location and thermal stability of implanted nickel in silicon studied by online emission channeling, J. Appl. Phys. 115, 023504 (2014).

[75] J. R. Tesmer and M. Nastasi, Handbook of Modern Ion Beam Materials Analysis (Materials Research Society, Pittsburgh, 1995). 